\title{
Transition from a strongly interacting 1D superfluid to a Mott insulator
}

\author{
Thilo Stöferle, Henning Moritz, Christian Schori, Michael Köhl ${ }^{\dagger}$ and Tilman Esslinger \\ Institute of Quantum Electronics, ETH Zürich Hönggerberg, CH-8093 Zürich, Switzerland
}

(Dated: October 16, 2003)

\begin{abstract}
We study one-dimensional trapped Bose gases in the strongly interacting regime. The systems are created in an optical lattice and are subject to a longitudinal periodic potential. Bragg spectroscopy enables us to investigate the excitation spectrum of the one-dimensional gas in different regimes. In the superfluid phase a broad continuum of excitations is observed which calls for an interpretation beyond the Bogoliubov spectrum taking into account the effect of quantum depletion. In the Mott insulating phase a discrete spectrum is measured. The excitation spectra of both phases are compared to the three-dimensional situation and to the crossover regime from one to three dimensions. The coherence length and coherent fraction of the gas in all configurations are measured quantitatively. We observe signatures for increased fluctuations which are characteristic for 1D systems. Furthermore, ceasing collective oscillations near the transition to the Mott insulator phase are found.

PACS numbers: 05.30.Jp, 03.75.Kk, 03.75.Lm, 73.43.Nq
\end{abstract}

Quantum gases trapped in the periodic potential of an optical lattice have opened a new experimental window on many-particle quantum physics. The recent observation of the quantum phase transition from a superfluid to a Mott insulating phase in a Bose gas [1] has offered a first glimpse on the physics which is now becoming experimentally accessible. However, the full wealth of possibilities has yet to be explored. Besides controlling the effect of interactions in the trapped gas, it is conceivable to induce disorder, to change the dimensionality of the system, or to trap Fermi gases or Bose-Fermi mixtures. The realization of these systems is expected to provide a deeper understanding of general concepts related to superfluidity and superconductivity.

Here we use the optical lattice to realize a strongly interacting Bose gas in one spatial dimension and to study the crossover to three dimensions. Emphasis is put on the measurement of excitation spectra which characterize the transition from the superfluid 2, 3] to the Mott insulating state [1, 4, 5]. Several features observed in the spectra go beyond the description of current theoretical models.

Degenerate Bose gases trapped in the lowest band of an optical lattice can be modelled using the Bose-Hubbard Hamiltonian [6, 7, 8, [9], in which the hopping of atoms between neighboring lattice sites is characterized by the tunnelling matrix element $J$, while the interaction energy for two atoms occupying the same site is given by $U$. The physics of this model is governed by the ratio between $U$ and $J$, i.e. between interaction and kinetic energy. This parameter can be controlled by changing the depth of the lattice potential. If the ratio $U / J$ is below a critical value the atoms are superfluid. Above this value the system becomes Mott insulating. We access the one-dimensional regime [6, 10, 11] using an anisotropic optical lattice consisting of three mutually perpendicular standing waves. By choosing large potential depths in two axes we can selectively suppress tunnelling and hopping is possible only along one dimension, so that an array of one-dimensional tubes with periodic modulation along their axis is formed.

We produce almost pure Bose-Einstein condensates of typically $1.5 \times 10^{5}{ }^{87} \mathrm{Rb}$ atoms in the $\left|F=2, m_{F}=2\right\rangle$ hyperfine ground state in a magnetic trap with trapping frequencies of $\omega_{x}=2 \pi \times 18 \mathrm{~Hz}, \omega_{y}=2 \pi \times 20 \mathrm{~Hz}$, and $\omega_{z}=$ $2 \pi \times 22 \mathrm{~Hz}$. The optical lattice is formed by three retroreflected laser beams which are derived from laser diodes at a wavelength of $\lambda=826 \mathrm{~nm}$ 12]. At the position of the condensate the beams are circularly focused to $1 / e^{2}$ radii of $120 \mu \mathrm{m}$ ( $x$ and $y$ axes) and $105 \mu \mathrm{m}(z)$. The three beams possess mutually orthogonal polarizations and their frequencies are offset with respect to each other by several ten MHz. The linewidth of the lasers is of the order of $10 \mathrm{kHz}$.

In order to load the condensate into the ground state of the optical lattice, the intensities of the lasers are slowly increased to their final values using an exponential ramp with a time constant of $25 \mathrm{~ms}$ and a duration of $100 \mathrm{~ms}$. The resulting optical potential depths $V_{x, y, z}$ are proportional to the laser intensities and are conveniently expressed in terms of the recoil energy $E_{R}=\frac{\hbar^{2} k^{2}}{2 m}$ with $k=\frac{2 \pi}{\lambda}$ and the atomic mass $m$. To prepare an array of one-dimensional tubes, two lattice axes are ramped to $V_{\perp} \equiv V_{x}=V_{z}=30 E_{R}$ and the third one to a much lower value $V_{a x, 0} \equiv V_{y}$. In this configuration the transverse tunnelling rates $J_{x}$ and $J_{z}$ are small and contribute a correction of the order of $J_{x, z} / \mu \ll 1$ to the 1 D characteristics of the individual tubes, where $\mu$ is the chemical potential of the sample. It is convenient to include the anisotropic tunnelling between all nearest neighbor sites which yields the resulting $J=2\left(J_{x}+J_{y}+J_{z}\right)$.

We study the excitation spectrum by employing amplitude modulation of the axial lattice potential $V_{a x}$ to perform two-photon Bragg spectroscopy [13]. The lattice potential takes the form $V_{a x}(y, t)=\left(V_{a x, 0}+\right.$ 
$\left.A_{\text {mod }} \sin \left(2 \pi \nu_{\text {mod }} t\right)\right) \sin ^{2}(k y)$. The modulation with amplitude $A_{\text {mod }}$ and frequency $\nu_{\text {mod }}$ introduces two sidebands with frequencies $\pm \nu_{\text {mod }}$ relative to the lattice laser frequency which define the energy $h \nu_{\text {mod }}$ of the excitation. Due to the Bragg condition atoms scattering two photons receive a momentum transfer of $0 \hbar k$ or $2 \hbar k$. In contrast to applying a potential gradient across the lattice [1], this method is not susceptible to effects like Bloch oscillations and Zener tunnelling which occur for low axial lattice depths. Furthermore, the excitation energy is precisely determined and does not involve any parameters that need calibration.

After the excitation, the experimental sequence continues by ramping down the lattice potentials linearly in $15 \mathrm{~ms}$ to $V_{a x}=V_{\perp}=4 E_{R}$ where the atoms are able to tunnel again in all three dimensions between the sites of the lattice. To allow for re-thermalization of the system, the atoms are kept at this lattice depth for $5 \mathrm{~ms}$. Then all optical and magnetic potentials are suddenly switched off. The resulting matter wave interference pattern is detected by absorption imaging after $25 \mathrm{~ms}$ of ballistic expansion. The width of the central momentum peak is taken as a measure of how much energy has been deposited in the sample by the excitation. If the energy increase is small, the peak is well fitted by a bimodal distribution. For resonant excitation there is only a single gaussian component, reflecting that the temperature of the atoms has significantly increased. To be independent of the shape of the peak we use the full width at half maximum (FWHM) as a measure of the introduced energy. Although this underestimates small energy increases, the important resonances and features of the spectra are well shown.

The duration $t_{\text {mod }}=30 \mathrm{~ms}$ and amplitude $A_{\text {mod }}=$ $0.2 V_{a x, 0}$ of the modulation are chosen such that the resulting excitation of the condensate does not exhibit saturation effects for all measurements presented here. We have verified that all atoms remain in the lowest Bloch band by adiabatically switching off the lattice potentials 14] after the modulation. When we load a cold thermal cloud into the lowest Bloch band and apply our modulation scheme, we do not observe excitations.

Figure1displays the fundamental change in the excitation spectrum for a 1D Bose gas when the crossover from the superfluid to the Mott insulating phase occurs: The broad continuum of the superfluid contrasts with the discrete spectrum of the Mott insulator. One surprising feature is that we can excite the superfluid with our scheme at large $h \nu_{\text {mod }}$ contrary to predictions for the weakly interacting superfluid in an optical lattice formed by a single standing wave [2]. In our experiment strong interactions lead to a significant quantum depletion, which is $\approx 50 \%$ for the $1 \mathrm{D}$ configuration even with $U / J=2.3[15]$. Therefore this parameter may not be regarded small as in standard Bogoliubov theory, but higher order excitations should be taken into account [16]. In combination

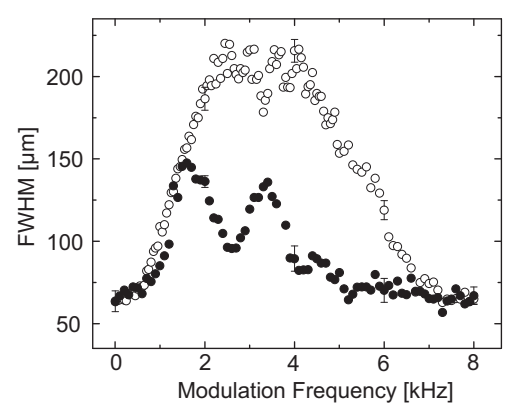

FIG. 1: Spectroscopy of the 1D superfluid (open circles) and the Mott insulating phase (filled circles) with values of $U / J$ of approximately 2.3 and 14 respectively. Error bars reflect the statistical error of 5 measurements.

with the broken translational invariance in the inhomogeneous trap, this could explain the non-vanishing excitation probability observed in the experiment at high energies [17.

A full series of spectra for different values of $U / J$ ranging from the superfluid via the crossover region to the Mott insulating phase is shown in figure 2 Figure 2a displays the $1 \mathrm{D}$ situation with $V_{\perp}=30 E_{R}$. The crossover from one to three dimensions (figure 2 b) is achieved by reducing the transverse confinement of the optical lattice to $V_{\perp}=20 E_{R}$. Then the tunnelling time between the $1 \mathrm{D}$ tubes is of the order of $t_{\text {mod }}$ and thus the interaction between the tubes is not negligible any more [18]. Finally, figure 2r shows the three-dimensional case with $V_{\perp}=V_{a x, 0}$.

In one dimension (figure $2 \mathrm{k}$ ) we observe the appearance of the discrete structure, which is characteristic for the Mott insulating phase, between $U / J=4$ and $U / J=8$. Above $U / J=20$ there is no more background due to the vanishing superfluid component. Our results are in accordance with the prediction $U / J=5.8$ for entering the $n=1$ Mott insulator based on a mean-field theory [ $[, 9]$. Calculations beyond the mean-field approach give an onset of the Mott insulating phase in the homogeneous 1D system at $U / J \approx 1.8[8]$. However, the finite size of the trap prohibits a sharp transition [10], so that the fraction of Mott insulating atoms increases gradually with $U / J$.

For the superfluid we obtain spectra which differ significantly from the results of ref. [1], since the superfluid excitations decrease at higher energies. This decrease is rather slow for the $1 \mathrm{D}$ gas but becomes more pronounced when the tunnelling between the 1D gases is increased (Figure 2 $2 \mathrm{~b}$ and Figure 2 ). Our excitation scheme does not induce dephasing that occurs when the strongly interacting condensate is accelerated near the edge of the Brillouin zone [19]. This might cause the broadening and the background in the tilted lattice experiments at high energies in ref. 1]. The width of the superfluid spectra for the 1D gas is on the same order as twice the width of the lowest band for Bogoliubov excitations [20]. 
a) $1 \mathrm{D}$

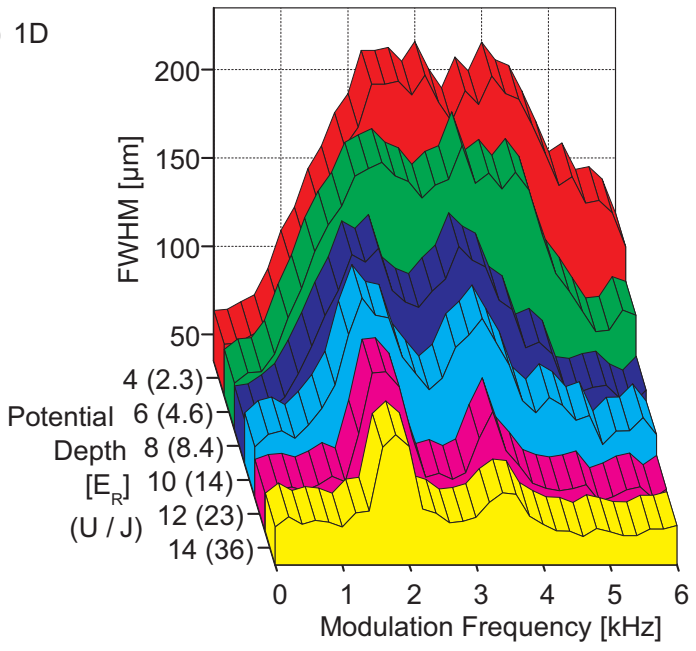

b) $1 \mathrm{D}-3 \mathrm{D}$

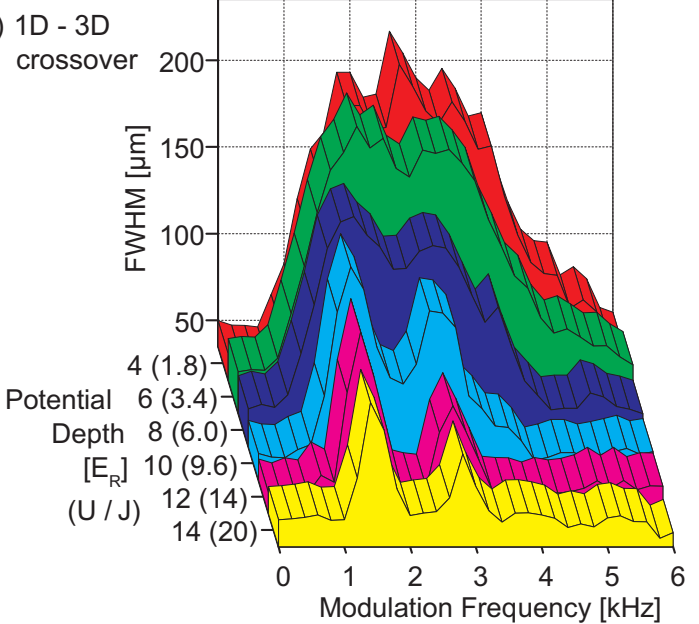

c) $3 \mathrm{D}$

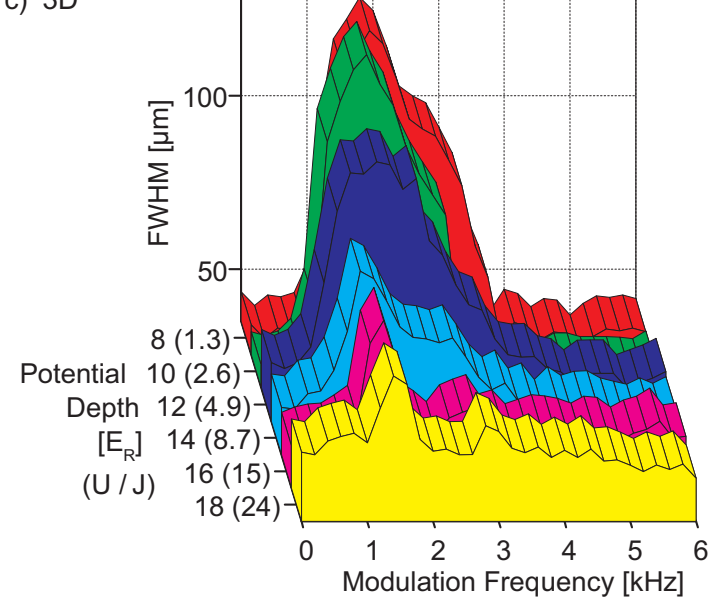

FIG. 2: The measured excitation spectrum of an array of 1D gases $\left(V_{\perp}=30 E_{R}\right)$ is shown in (a) for different values of $V_{a x, 0}$. The interaction ratios $U / J$ given in brackets are calculated numerically using a band structure model in the tightbinding approximation [9]. Spectrum (c) shows the superfluid to Mott insulator transition in the $3 \mathrm{D}$ case $\left(V_{\perp}=V_{a x, 0}\right)$. The crossover region between the one- and the three-dimensional system $\left(V_{\perp}=20 E_{R}\right)$ is shown in (b).
In the Mott insulating phase we find the first resonant peak for all data sets close to the calculated value of $U$. A second peak appears at $(1.91 \pm 0.04)$ times the energy of the first resonance, somewhat smaller than the value of 2 reported in [1]. This resonance might be attributed to defects where lattice sites with $n=1$ atom next to sites with $n=2$ atoms are being excited. For the 1D system and in the dimensional crossover regime (Fig. 22a and b) a much weaker resonance appears at $(2.60 \pm 0.05)$ times the energy of the first resonance which could indicate higher order processes of two atoms tunnelling simultaneously. In figure $3 \mathrm{k}$ we plot the rms width of the first resonance in the excitation spectrum of the Mott insulating phase when fitted by a gaussian. In figure Bb we show the ratio of the amplitudes of the second and the first peak. Apparently, in the 1D system the first peak is wider and the second peak more pronounced as compared to the 3D situation, which could be an indication of increased fluctuations in $1 \mathrm{D}$ systems.

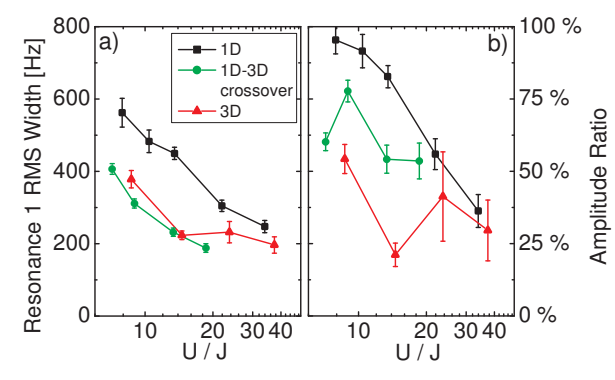

FIG. 3: (a) Width of the first resonance peak in the spectrum of the Mott insulator. (b) Ratio of the amplitudes of the second and the first peak of the spectrum of the Mott insulator. The error bars mark the error of the gaussian fits.

Compared to the superfluid properties the coherence properties of the system provide complementary information about the state of the gas. They are probed by studying the matter wave interference pattern 1, 21]. Here we first prepare the array of 1D systems as above but do not apply our excitation scheme. Instead, after holding the atoms at the final lattice depth for $t_{h}=$ $30 \mathrm{~ms}$, we increase $V_{a x}$ rapidly $(<40 \mu \mathrm{s})$ to about $25 E_{R}$ and then abruptly switch off all optical and magnetic trapping potentials. This procedure projects the different initial configurations onto the same Bloch state. To extract the number of coherent atoms $N_{c o h}$ from the interference pattern, the peaks [23] at $0 \hbar k, \pm 2 \hbar k$ and $\pm 4 \hbar k$ are fitted by gaussians (Fig. 4 ). Incoherent atoms give rise to a broad gaussian background which dominates for higher $V_{a x, 0}$. Taking this fit as a measure of the number of incoherent atoms $N_{\text {incoh }}$, we calculate the coherent fraction $f_{c}=\frac{N_{c o h}}{N_{c o h}+N_{\text {inco }}}$. As shown in figure $4 \mathrm{a}, f_{c}$ decreases slowly to zero for increasing values of $U / J$ and appears almost independent of the dimensionality. This coincides with the prediction that for strongly interacting Bose gases in optical lattices the superfluid fraction 
can be significantly different from the coherent fraction, and that the decrease of $f_{c}$ is not a sufficient signature of entering the Mott insulating phase 22]. In figure 45 we plot the width of the central peak of the interference pattern, which is a measure of the coherence length of the gas. An increasing width is a good indicator for the presence of a Mott insulating phase since even a small Mott insulating domain reduces the coherence length of the sample, as elucidated in numerical calculations [24]. Our data shows that the increase in width starts at much lower values of $U / J$ for the $1 \mathrm{D}$ gas than for the $3 \mathrm{D}$ gas. This supports the expectation that due to the more pronounced quantum fluctuations in the 1D geometry the gas enters the Mott insulating state at lower values of $U / J$ 8, 10, 11, 24]. Experimentally, thermal fluctuations may also contribute to the observed width. However, by comparing the width of the interference peak for different hold times $\left(t_{h}=1 \mathrm{~ms}\right.$ and $\left.t_{h}=30 \mathrm{~ms}\right)$ we find that the primary effect of the additional heating is an overall increase of the width rather than a change of the slope of the curve.
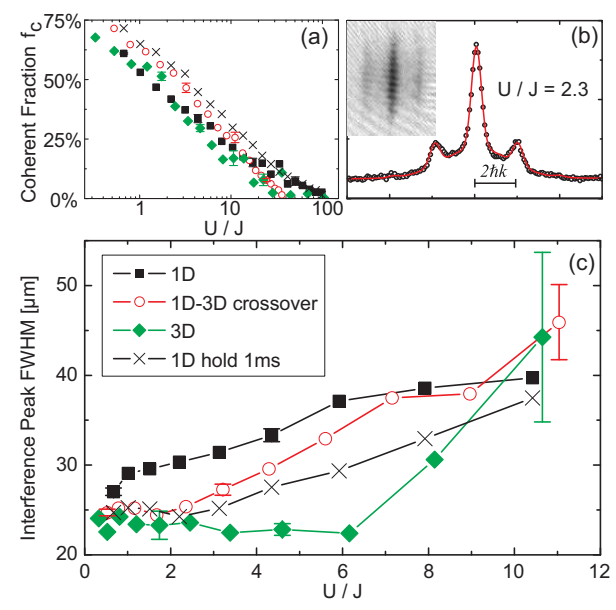

FIG. 4: (a) Coherent fraction vs. $U / J$. The error bars are determined by the statistical error of 4 measurements. (b) The column sum of the optical density (circles) and the fits (solid line) from which the number of coherent and incoherent atoms are deduced for the 1D case. The inset shows the absorption image after $10 \mathrm{~ms}$ of time-of-flight (dimensions $360 \mu \mathrm{m} \times 467 \mu \mathrm{m}$ ). (c) Width of the central momentum peak vs. $U / J$.

One feature of the Mott insulating phase is its lack of compressibility [ $\underline{6}$ ]. If a potential gradient is applied across the lattice, the inability to redistribute atoms in the lattice results in a breakdown of the flow of atoms. Even if only a fraction of the atoms in our inhomogeneous trap is locally incompressible and pinned in the Mott insulating state [10], collective oscillations should suspend. In the experiment, the array of one-dimensional gases is prepared in the same way as before. Then, we apply a small potential gradient (far less than any resonance gradients [1]) for $1 \mathrm{~ms}$ to accelerate the atoms in the axial direction. We observe a very strong increase in the damping of the small amplitude dipole oscillations even for very low $V_{a x, 0}$, a behaviour which is different from the three-dimensional gas. Critical damping is reached at $V_{a x, 0}=3 E_{R}$, and at $V_{a x, 0}=10 E_{R}$ we can not displace the atom cloud any more.

In conclusion, we have prepared an array of onedimensional strongly interacting Bose gases and measured the excitation spectra in the superfluid and the Mott insulating regime. The spectrum of the superfluid exhibits excitations at high energies which are not predicted by current theories for weakly interacting bosons in an optical lattice and have so far only been discussed in the context of superfluid Helium. This shows that the strong interactions and the significant quantum depletion change the properties of the Bose gas considerably, already much below the crossover to the Mott insulator. The effect of reduced dimensionality on the crossover from superfluid to Mott insulator was most pronounced in the measurements of the coherence length of the gas.

We would like to thank H. P. Büchler and G. Blatter for insightful discussions, and SNF and QSIT for funding.

[†] email:Koehl@iqe.phys.ethz.ch

[1] M. Greiner et al., Nature 415, 39 (2002).

[2] C. Menotti, M. Krämer, L.Pitaevskii, S. Stringari, Phys. Rev. A 67, 053609 (2003).

[3] A. M. Rey et al., J. Phys. B 36, 825 (2003).

[4] K. Sheshadri, H.R. Krishnamurthy, R. Pandit ,T.V. Ramakrishnan, Europhys. Lett. 22, 257 (1993).

[5] S. Sachdev, K. Sengupta, S.M. Girvin, Phys. Rev. B 66, 075128 (2002).

[6] M. P. A. Fischer, P.B. Weichmann, G. Grinstein, D.S. Fisher, Phys. Rev. B 40, 546 (1989).

[7] J. K. Freericks, H. Monien, Europhys. Lett. 26, 545 (1994).

[8] T. D. Kühner, H. Monien, Phys. Rev. B 58, 14741 (1998).

[9] D. Jaksch et al., Phys. Rev. Lett. 81, 3108 (1998).

[10] G. G. Batrouni et al., Phys. Rev. Lett. 89, 117203 (2002).

[11] H. P. Büchler, G. Blatter, W. Zwerger, Phys. Rev. Lett. 90, 130401 (2003).

[12] H. Moritz, T. Stöferle, M. Köhl, T. Esslinger, e-print cond-mat/0307607

[13] J. Stenger et al., Phys. Rev. Lett. 82, 4569 (1999).

[14] M. Greiner et al., Phys. Rev. Lett. 87, 160405 (2001).

[15] M. Krämer, C. Menotti, L. Pitaevskii, S. Stringari, Eur. Phys. J. D 27, 247 (2003).

[16] N.M. Hugenholtz, D. Pines, Phys. Rev. 116, 489 (1959).

[17] H. P. Büchler, private communication.

[18] A. F. Ho, M. A. Cazalilla, T.Giamarchi, e-print cond-mat/0310382

[19] J. C. Bronski, L.D. Carr, B. Deconinck, J.N. Kutz, Phys. Rev. Lett. 86, 1402 (2001).

[20] M. Krämer, private communication.

[21] C. Orzel et al., Science 291, 2386 (2001).

[22] R. Roth, K. Burnett, Phys. Rev. A 67, 031602 (2003). 
[23] At $V_{\perp}=30 E_{R}$, coherence between the individual 1D systems is lost after a few milliseconds [12]. Perpendicular to the axis of the tubes the expansion of the ground state is gaussian.
[24] C. Kollath, U. Schollwöck, J. von Delft, W. Zwerger, eprint cond-mat/0310388 